\title{
Rituximab Therapy for Severe Cutaneous Leukocytoclastic Angiitis Refractory to Corticosteroids, Cellcept and Cyclophosphamide
}

\author{
Kamel El-Reshaid ${ }^{a} \quad J o h n$ Patrick Madda ${ }^{b}$ \\ ${ }^{a}$ Department of Medicine, Faculty of Medicine, Kuwait University, and \\ ${ }^{b}$ Department of Histopathology, Al-Amiri Hospital, Kuwait City, Kuwait
}

\section{Key Words}

Cutaneous vasculitis $\cdot$ Cyclophosphamide $\cdot$ Rituximab

\begin{abstract}
We report our clinical experience with rituximab in the treatment of 2 patients with idiopathic cutaneous angiitis who relapsed after treatment with high-dose corticosteroids and cyclophosphamide. A 39-year-old woman and a 51-year-old man presented with ulcerating maculopapular rash in both lower limbs which relapsed 6 months after treatment with a combination of high-dose corticosteroids and cyclophosphamide. After treatment with $2 \mathrm{~g}$ of rituximab, the first patient has still been in clinical remission for 32 months while the second has finished 28 months. Interestingly, CD19 which had dropped to $<0.1$ one week following the start of retuximab had increased to $>0.5 \% 8$ months later in both patients. Despite that, our patients are still in clinical remission. No significant side effects were noted during infusions and up to the period of follow-up. In conclusion, rituximab is a useful and safe agent in the treatment of idiopathic cutaneous angiitis refractory to conventional therapy. Clinical remission persists years after improvement of B-cell suppression.
\end{abstract}

\section{Introduction}

Cutaneous leukocytoclastic angiitis (CLA) can be a manifestation of systemic vasculitis, HSP, hypersensitivity to drugs, serum, infection, HIV or idiopathic and isolated [1]. Idiopathic CLA was classified as microscopic polyangiitis since these leukocytoclastic changes are pauci-immune and seldom seen without isolated microscopic hematuria [2]. Hence, skin

Prof. Dr. Kamel El-Reshaid

Department Of Medicine, Faculty of Medicine

Kuwait University, PO Box 24923

Safat 13110 (Kuwait)

E-Mail Kamelelreshaid@yahoo.com 
involvement is limited to the subepidermal vessels and kidney involvement is limited to small venules rather than small arterioles or medium-sized ones seen in microscopic polyangiitis or polyarteritis nodosa that can be detected by kidney biopsy and/or celiac and renal arteriography [2]. The pattern of cutaneous pathology and immunopathology is highly sensitive to the timing of the biopsy and the age of the lesion. Lesions that are less than 24 hours old are more likely to reveal leukocytoclastic changes and are polymorphonuclear predominant, while older lesions show enhanced numbers of mononuclear cells [3]. Little correlation exists between the clinical manifestations and the specific histological pattern [4]. The natural history of CLA is unclear. Progressive and devastating skin-limited lesions have been described, yet minor skin changes may pass unrecognized or being reported as part of the systemic disease. Secondary forms may be amenable to discontinuation of the offending drug, serum or treatment of hepatitis and sepses. However, data on treatment of idiopathic form is lacking. Colchicine, antihistaminics, dapsone, pentoxifylline or combinations of these drugs have been tried $[1,5,6]$. However, severe and progressive or recurrent CLA refractory to these agents and those for systemic vasculitis such as corticosteroids and cyclophosphamide are rarely reported. Herein, we report on our experience with the use of rituximab in this field.

\section{Case Reports}

Case 1

A 39-year-old Lebanese woman presented with progressive skin rash with ulcerations in both lower limbs for 2 months. She denied fever, weight loss, chest or abdominal pain. She had mild low back discomfort at the left sacroiliac region, yet no peripheral joint pain or swelling. The patient is a school teacher and had never dealt with paints or lived in an area of industrial fumes. She did not have previous medical disease, allergy, and surgery nor had used medications especially in the past few months prior to the skin rash. On her initial physical examination, the patient was in clear discomfort from her leg ulcers. Blood pressure was 110/70 mm Hg, temperature was normal and body weight was $70 \mathrm{~kg}$. She did not have lymphadenopathy, goiter, jugular venous distension or edema. Systemic examination did not show abnormality. Lower extremities showed bilateral maculopapular rash with ulcerations and livida reticularis (fig. 1).

Laboratory investigations showed normal peripheral leukocytic and platelet counts. Hemoglobin was $110 \mathrm{~g} / \mathrm{l}$ with normal MCV. ESR was $90 \mathrm{~mm} / \mathrm{h}$. Serum sugar, urea, creatinine, electrolytes, CPK, liver functions, cholesterol and TSH were normal. Urine routine and microscopy was normal except for isolated hematuria (25 RBCs/HPF). Blood culture was negative. Serum complements (C3 and C4) and protein electrophoresis were normal. ANA, anti-ds DNA, ANCA, RA, cryoglobulins, hepatitis B surface antigen and anti-HCV antibodies were negative. Twenty-four-hour urine collection showed normal creatinine clearance and protein excretion. Stool testing for ova, parasites and occult blood was normal. Chest X-ray and ECG were normal. Abdominal and pelvic ultrasound did not show abnormality. Skin biopsy showed leukocytoclastic vasculitis with predominantly lymphocytic infiltrate in the subepidermal layer only with extravasated fragmented erythrocytes indicating vascular leaks (fig. 2). Immunofluorescent stains were negative. Since she had hematuria, percutaneous kidney biopsy as well as celiac and renal arteriography were done. Both tests were normal. The patient was previously treated with 6 weeks of corticosteroids $(1 \mathrm{mg} / \mathrm{kg} /$ day $)$ but the lesions persisted. Hence, she was treated with pulse steroids (1 g i.v. daily for 3 days) followed by prednisone oral corticosteroids in addition to cyclophosphamide monthly 
infusions at a dose of $1 \mathrm{~g}$ in $200 \mathrm{ml}$ of normal saline over $2 \mathrm{~h}$. The latter was continued for 3 months, then was reduced to every 3 months with a maintenance daily dose of $5 \mathrm{mg}$ of prednisone for a total period of 18 months. The lesions disappeared after the first month of therapy. However, 6 months after discontinuation of cyclophosphamide therapy, her lesions reappeared. Again, systemic examination and investigations did not show evidence of systemic disease. At this time, she was treated with 4 weekly infusions of rituximab (mabthera). The patient was premedicated with two 500-mg paracetamol and one piriton tablet followed by an infusion of $125 \mathrm{mg}$ of solumedrol in $50 \mathrm{ml}$ of D5W over $30 \mathrm{~min}$ before infusions. The $500 \mathrm{mg}$ of rituximab were diluted in $450 \mathrm{ml}$ of NS leading to a concentration of $1 \mathrm{mg} / \mathrm{ml}$. The first infusion rate was $20 \mathrm{ml} / \mathrm{h}$ for the first $30 \mathrm{~min}$ followed by $20-\mathrm{ml}$ increments/h every $30 \mathrm{~min}$ till the total dose is achieved. The second infusion was started at $40 \mathrm{ml} / \mathrm{h}$, the third at $80 \mathrm{ml} / \mathrm{h}$ and the fourth at $120 \mathrm{ml} / \mathrm{h}$. Flow cytometry showed that her CD19 lymphocytes dropped to zero after 1 week of therapy and remained $<0.5 \%$ for 8 months. Interestingly, the patient is asymptomatic and up to 12 months after normalization of her CD19, i.e. a total of 32 months following the start of rituximab therapy.

Case 2

A 51-year-old Kuwaiti man was referred to our renal unit for similar skin lesions and isolated hematuria which had developed over a few weeks. He received a 6-week course of oral corticosteroids $(1 \mathrm{mg} / \mathrm{kg} /$ day) without improvement. He was subjected to the same systematic assessment in the first patient which had included routine tests, serology as well as skin and kidney biopsy and celiac and renal arteriography. Skin biopsy confirmed the diagnosis of CLV with negative immune deposits and kidney biopsy was normal. He was initially treated with pulse medrol and cyclophosphamide as the previous patient and responded. In a similar fashion, he relapsed 6 months after discontinuation of the 12-month course. His lesions regressed with cellcept $1 \mathrm{~g}$ twice daily. However, they flared once the dose was reduced to $500 \mathrm{mg}$ twice daily. After 6 months of such therapy, he received rituximab as in the previous protocol. He responded well and up to 16 months post-therapy. In a similar fashion, his CD19 remained $<0.5 \%$ for 8 months following retuximab treatment then normalized, yet he still enjoys a good clinical remission 28 months since the start of therapy.

\section{Discussion}

Rituximab is a chimeric human/mouse monoclonal antibody that is approved, in the USA since 1997, for the treatment of non-Hodgkin's lymphoma and recently for severe rheumatoid arthritis [7]. The drug binds avidly to the CD20 antigen expressed on normal differentiated B-lymphocytes and pre-B-cells, but not stem cells or plasma cells. Unlike murine monoclonal antibodies to CD20, rituximab persists in the circulation for long periods, interacts with human effector cells and rarely generates human anti-mouse antibody responses. It destroys B-cells by multiple mechanisms including complementdependent cytotoxicity, antibody-dependent cellular cytotoxicity, induction of apoptosis and sensitization to other chemotherapeutic agents [8]. This potent chemotherapeutic effect was associated with good tolerability profile with mild to moderate infusion-related reactions and hence has to be administered slowly over hours especially at the first time [7].

The drug has shown potential in the treatment of many autoimmune diseases including systemic vasculitis and lupus erythematosus [9]; hence it was used in our 2 cases with refractory CLA. In 2006, Chung et al. [10] used rituximab to treat 2 cases of severe and 
El-Reshaid et al.: Rituximab Therapy for Severe Cutaneous Leukocytoclastic Angiitis Refractory to Corticosteroids, Cellcept and Cyclophosphamide

progressive cutaneous vasculitis. The first was a transplant patient who presented with skin lesions and renal failure. The etiology of the renal disease in his native kidneys was uncertain, yet biopsy of his transplanted kidney failed to show vasculitis. Treatment with rituximab not only improved his $\mathrm{CV}$ but also his renal impairment. The second case was a patient who had received treatment for non-Hodgkin's lymphoma 15 years before. She presented with lesions and computed tomography showed inguinal and mediastinal lymphadenopathy. Moreover, positron emission tomography demonstrated hypermetabolic lymph nodes within the subcarinal and perihilar regions. Subsequent mediastinoscopy with lymph node biopsy showed paracortical hyperplasia with monocytoid B-cell hyperplasia, and an excess of plasma cells. However, the findings of a bone marrow biopsy were unremarkable for lymphoma recurrence. The patient responded to therapy. Whether their 2 cases represent secondary forms of vasculitis, viz. systemic vasculitis in the first patient and paraneoplastic manifestation of early non-Hodgkin's lymphoma in the second, rather than idiopathic CLA is unclear. On the other hand, our 2 cases represent clear cases of idiopathic CLA with lack of secondary etiology established by their clinical presentation, laboratory and radiological findings as well as their definite limitation to subepidermal layer by skin histology [2, 3], and are hence worth reporting. Furthermore, both patients presented with a disease which is refractory to corticosteroids and cyclophosphamide, yet were safely treated with retuximab. Interestingly, the clinical remission induced by rituximab persisted beyond the effect of B-cell suppression induced by the drug as CD19 normalized after 8 months posttherapy. The latter phenomenon may be related to alteration in the memory cells which may persist up to 6 years $[11,12]$.

In conclusion, our case report describes 2 cases with clear idiopathic CLA. As described, the disease can be associated with hematuria due to venular disease. It can be recurrent and refractory to the available potent immunosuppressive agents such as cyclophosphamide up to 2 years of therapy. Fortunately, rituximab can be a useful alternative.

\section{References}

1 Blanco R, Martinez-Taboada VM, Rodriguez-Valverede V, Garcia-Fuentes M: Cutaneous vasculitis in children and adults: Associated diseases and etiologic factors in 303 patients. Medicine (Baltimore) 1998;77:403418.

2 Jennette JC, Falk RJ, Andrassy K, Bacon PA, Churg J, Gross WL, Hagen EC, Hoffman GS, Hunder GG, Kallenberg CG: Nomenclature of systemic vasculitides: Proposal of an international consensus conference. Arthritis Rheum 1994;37:187-192.

-3 Zax RH, Hodge SJ, Callen JP: Cutaneous leukocytoclastic vasculitis. Arch Dermatol 1990;126:69-72.

4 Mullick FG, McAlister HA, Wagner BM, Fenoglio JJ Jr: Drug-related vasculitis: Clinicopathologic correlations in 30 patients. Hum Pathol 1979;10:313-325.

5 Sais G, Vidaller A, Jucgla A, Gallardo F, Peyri J: Colchacine in the treatment of cutaneous leukocytoclastic vasculitis. Results of a prospective, randomized controlled trial. Arch Dermatol 1995;131:1399-1402.

-6 Nurnberg W, Grabbe J, Czarnetzki BM: Urticarial vasculitis syndrome effectively treated with dapsone and pentoxifylline. Acta Derm Venereol 1995;75:54-56.

7 Kimby E: Tolerability and safety of retuximab (MabThera). Cancer Treat Rev 2005;31:456-473.

8 Johnson P, Glennie M: The mechanism of action of rituximab in the elimination of tumor cells. Semin Oncol 2003;30(suppl 2):2-8.

-9 El-Halak M, Binstadt BA, Leichtener AM, Bennett CM, Neufeld EJ, Fuhlbrigge RC, Zurakowski D, Sundel RP: Clinical effects and safety of rituximab for treatment of refractory pediatric autoimmune diseases. J Pediatr 2007;150:376-382.

10 Chung L, Funke AA, Chkravarty EF, Callen JP, Fiorentino DF: Successful use of rituximab for cutaneous vasculitis. Arch Dermatol 2006;142:1407-1410. 


\section{Case Reports in Dermatology}

\begin{tabular}{l|l}
\hline Case Rep Dermatol 2013;5:115-119 \\
\hline DOI: 10.1159/000350559 & $\begin{array}{l}\text { C 2013 S. Karger AG, Basel } \\
\text { www.karger.com/cde }\end{array}$ \\
\hline
\end{tabular}

El-Reshaid et al.: Rituximab Therapy for Severe Cutaneous Leukocytoclastic Angiitis Refractory to Corticosteroids, Cellcept and Cyclophosphamide

11 Muhammad K, Roll P, Einsele H, Dorner T, Tony HP: Delayed acquisition of somatic hypermutations in repopulated IgD+CD27+ memory B cell receptors after rituximab treatment. Arthritis Rheum 2009;60:2284-2293.

12 Snider RA, Book BK, Agarwal A, Bearden CM, Vieira CA, Pescovitz MD: In vivo human B-cell subset recovery after in vivo depletion with rituximab, anti-human CD20 monoclonal antibody. Hum Antibodies 2004;13:55-62.
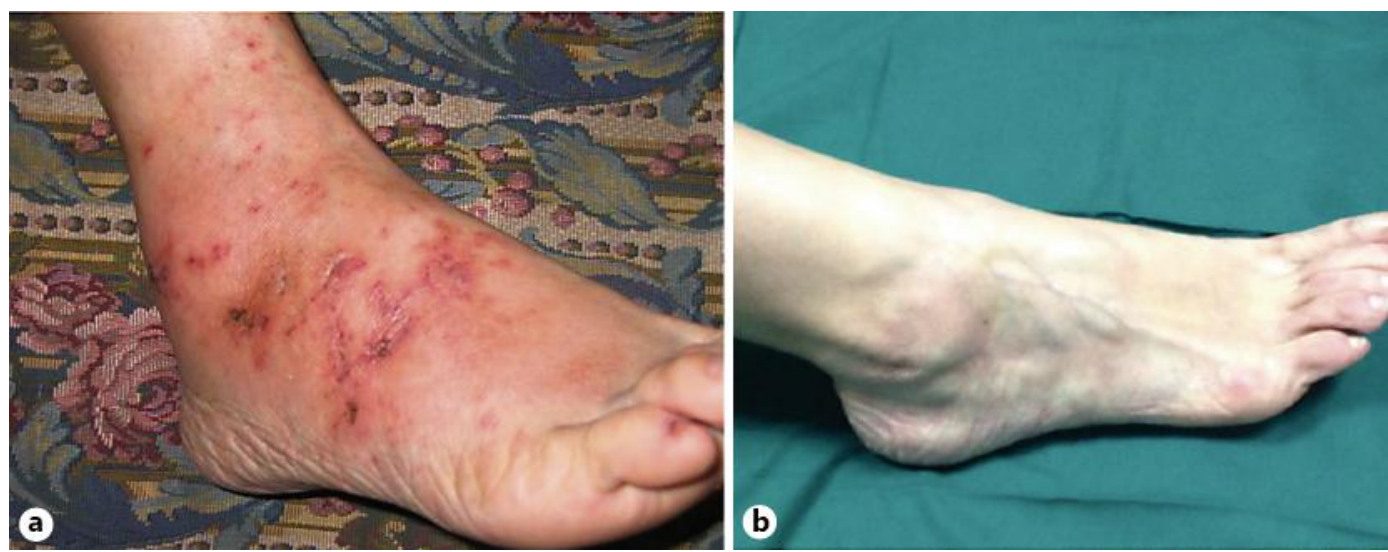

Fig. 1. a Skin lesions in patient 1 which consisted of maculopapular rash with ulcerations and edema at lower leg and foot before treament. $\mathbf{b}$ After treatment with rituximab.

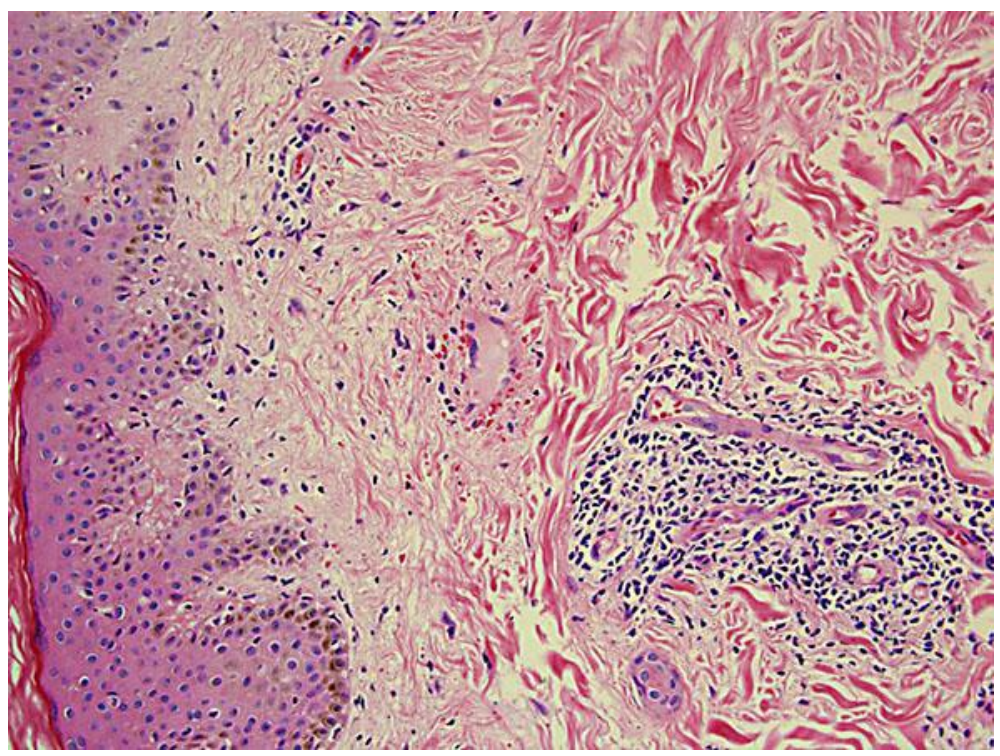

Fig. 2. Photomicrograph of a section of skin in patient 1, showing dilated subepidermal small vessels with swollen endothelial cells. There is a perivascular infiltrate consisting mainly of lymphocytes, few plasma cells and extravasated erythrocytes of which some are fragmented. No vascular necrosis is seen (HE, $\times 200$ ). 